Dehgardi N. ${ }^{1}$, SheikHOleslami S.M. ${ }^{2}$, SOROUdi M. ${ }^{2}$

\title{
SOME DISTANCE BASED INDICES OF GRAPHS BASED ON FOUR NEW OPERATIONS RELATED TO THE LEXICOGRAPHIC PRODUCT
}

For a (molecular) graph, the Wiener index, hyper-Wiener index and degree distance index are defined as $W(G)=\sum_{\{u, v\} \subseteq V(G)} d_{G}(u, v), W W(G)=W(G)+\sum_{\{u, v\} \subseteq V(G)} d_{G}(u, v)^{2}$, and $D D(G)=$ $\sum_{\{u, v\} \subseteq V(G)} d_{G}(u, v)(d(u / G)+d(v / G))$, respectively, where $d(u / G)$ denotes the degree of a vertex $u$ in $G$ and $d_{G}(u, v)$ is distance between two vertices $u$ and $v$ of a graph $G$. In this paper, we study Wiener index, hyper-Wiener index and degree distance index of graphs based on four new operations related to the lexicographic product, subdivision and total graph.

Key words and phrases: Wiener index, degree distance index, hyper-Wiener index, lexicographic product, subdivision, total graph.

\footnotetext{
${ }^{1}$ Department of Mathematics and Computer Science, Sirjan University of Technology, 7813733385, Sirjan, Iran

2 Department of Mathematics, Azarbaijan Shahid Madani University, 5375171379, Tabriz, Iran

E-mail: n.dehgardiesirjantech.ac.ir(Dehgardi N.),

s.m.sheikholeslami@azaruniv.ac.ir(Sheikholeslami S.M.),

m. soroudieazaruniv.ac.ir (Soroudi M.)
}

\section{INTRODUCTION}

In this paper $G$ is a simple and connected graph with vertex set $V=V(G)$ and edge set $E=$ $E(G)$. The degree of a vertex $v$ in $G$ is the number of edges incident to $v$ and denoted by $d(v / G)$. The distance $d_{G}(u, v)$ between any two vertices $u$ and $v$ of a graph $G$ is equal to the length of a shortest path connecting them. A line graph, $L(G)$, is the graph whose vertices correspond to the edges of $G$ and two vertices of $L(G)$ are adjacent if and only if the corresponding edges in $G$ are adjacent.

In chemical graph theory, a graphical invariant is a number related to a graph which is structurally invariant. These invariant numbers are also known as the topological indices. The well-known Zagreb indices are one of the oldest graph invariants firstly introduced by Gutman and Trinajstić [18], where they examined the dependence of total $\pi$-electron energy on molecular structures, and this was elaborated on in [17]. For a (molecular) graph $G$, the first Zagreb index $M_{1}(G)$ and the second Zagreb index $M_{2}(G)$, are:

$$
M_{1}(G)=\sum_{v \in V(G)} d(v / G)^{2}=\sum_{u v \in E(G)}[d(u / G)+d(v / G)]
$$

and

$$
M_{2}(G)=\sum_{u v \in E(G)} d(u / G) d(v / G) .
$$

$\mathrm{y}_{\Delta} \mathrm{K} 519.17$

2010 Mathematics Subject Classification: 05C07, 05C12, 05 C76.

Corresponding author: Dehgardi N. 
For properties of the two Zagreb indices see [4-6] and the papers cited therein. In recent years, some novel variants of Zagreb indices have been put forward, such as Zagreb coindices $[2,10,15]$, reformulated Zagreb indices [20,24], Zagreb hyper index [3, 25], multiplicative Zagreb indices [13,30], multiplicative sum Zagreb index [11,28], and multiplicative Zagreb coindices [29], etc. The Zagreb coindices are defined as:

$$
\overline{M_{1}}(G)=\sum_{u v \notin E(G)}[d(u / G)+d(v / G)]
$$

and

$$
\overline{M_{2}}(G)=\sum_{u v \notin E(G)} d(u / G) d(v / G) .
$$

The Wiener index of $G$ is denoted by $W(G)$ and is defined by

$$
W(G)=\sum_{\{u, v\} \subseteq V(G)} d_{G}(u, v) .
$$

The name Wiener index or Wiener number for the quantity defined in Equation (1) is usual in chemical literature, since Harold Wiener [27] in 1947 seems to be the first who considered it. Wiener himself conceived W only for acyclic molecules and defined it in a slightly different-yet equivalent-manner; the definition of the Wiener index in terms of distances between vertices of a graph, such as in Equation (1), was first given by Hosoya [19]. Eliasi et. al [12], determined the Wiener index of some graph operations.

The hyper-Wiener index of $G$ is denoted by $W W(G)$, and is defined as

$$
W W(G)=W(G)+\sum_{\{u, v\} \subseteq V(G)} d_{G}(u, v)^{2} .
$$

Lukovits [23] derived formulas for the hyper-Wiener index of chains and trees which contain one trivalent or tetravalent branching vertex, and this index is studied by several authors in $[1,8,16,22]$. Khalifeh et. al [21], determined the hyper-Wiener index of graph operations.

The degree distance of a graph $G, D D(G)$, was introduced by Dobrynin and Kochetova [9] and Gutman [14] as a weighted version of the Wiener index, and is defined as

$$
D D(G)=\sum_{\{u, v\} \subseteq V(G)} d_{G}(u, v)(d(u / G)+d(v / G)) .
$$

In this paper, we study of the Wiener, hyper-Wiener and degree distance indices of graphs based on operations related to the lexicographic, subdivision and total graph. For this purpose, we recall some operations on graphs in the following.

The composition or lexicographic product of two connected graphs $G_{1}$ and $G_{2}$, denoted by $G_{1}\left[G_{2}\right]$, is a graph with vertex set $V\left(G_{1}\right) \times V\left(G_{2}\right)$ and two vertices $u=\left(u_{1}, v_{1}\right)$ and $v=\left(u_{2}, v_{2}\right)$ of $G_{1}\left[G_{2}\right]$ are adjacent if and only if either $u_{1}$ is adjacent to $u_{2}$ or $u_{1}=u_{2}$ and $v_{1}$ is adjacent with $v_{2}$. For a connected graph $G$, there are four related graphs as follows:

(i) $S(G)$ is the graph obtained by inserting an additional vertex in each edge of $G$. Equivalently, each edge of $\mathrm{G}$ is replaced by a path of length 2;

(ii) $R(G)$ is the graph obtained from $G$ by adding a new vertex corresponding to each edge of $G$ and joining each new vertex to the end vertices of the corresponding edge; 
(iii) $Q(G)$ is the graph obtained from $G$ by inserting a new vertex into each edge of $G$ and joining those pairs of new vertices on adjacent edges of $G$;

(iv) $T(G)$ is the graph with vertex set $V(G) \cup E(G)$ and adjacency in $T(G)$ is defined as adjacency or incidence for the corresponding elements of $G$.

The graphs $S(G)$ and $T(G)$ are called the subdivision graph and the total graph of $G$, respectively.

Based on the lexicographic product of two connected graphs $G_{1}$ and $G_{2}$, Sarala et al. [26], introduced four new operations on these graphs.

Let $F \in\{S, R, Q, T\}$. The $F$-product of $G_{1}$ and $G_{2}$, denoted by $G_{1}\left[G_{2}\right]_{F}$, is defined by $F\left(G_{1}\right)\left[G_{2}\right]-E^{*}$, where $E^{*}=\left\{\left(u, v_{1}\right)\left(u, v_{2}\right) \in E\left(F\left(G_{1}\right)\left[G_{2}\right]\right): u \in V\left(F\left(G_{1}\right)\right)-V\left(G_{1}\right)\right.$ and $\left.v_{1} v_{2} \in E\left(G_{2}\right)\right\}$, i.e., $G_{1}\left[G_{2}\right]_{F}$ is a graph with the vertex set $V\left(G_{1}\left[G_{2}\right]_{F}\right)=\left(V\left(G_{1}\right) \cup E\left(G_{1}\right)\right) \times$ $V\left(G_{2}\right)$ and two vertices $u=\left(u_{1}, v_{1}\right)$ and $v=\left(u_{2}, v_{2}\right)$ of $G_{1}\left[G_{2}\right]_{F}$ are adjacent if and only if either $\left[u_{1}=u_{2} \in V\left(G_{1}\right)\right.$ and $\left.v_{1} v_{2} \in E\left(G_{2}\right)\right]$ or $\left[u_{1} u_{2} \in E\left(F\left(G_{1}\right)\right)\right.$ and $\left.v_{1}, v_{2} \in V\left(G_{2}\right)\right]$.

Sarala et al. [26] determined the Zagreb indices of $F$-product of $G_{1}$ and $G_{2}$ where $F \in$ $\{S, R, Q, T\}$, and Dehgardi et. al [7] computed the leap Zagreb indices of these graphs.

We will use the following results.

Theorem 1 ([7]). Let $G_{1}$ and $G_{2}$ be two connected graphs, and let $G=G_{1}\left[G_{2}\right]_{F}$ be the F-product of $G_{1}$ and $G_{2}$. Then

$$
d_{G}((u, x),(v, y))= \begin{cases}1 & \text { if } u=v \in V\left(G_{1}\right), x y \in E\left(G_{2}\right) \\ 2 & \text { if } u=v \in V\left(G_{1}\right), x y \notin E\left(G_{2}\right) \\ 2 & \text { if } u=v \in V\left(F\left(G_{1}\right)\right)-V\left(G_{1}\right) \\ d_{F\left(G_{1}\right)}(u, v) & \text { if } u \neq v .\end{cases}
$$

Theorem 2 ([15]). Let $G$ be a graph with $n$ vertices and $m$ edges. Then

$$
M_{1}(G)+\overline{M_{1}}(G)=2 m(n-1) .
$$

Theorem 3 ([15]). Let $G$ be a graph with $n$ vertices and $m$ edges. Then

$$
M_{2}(G)+\overline{M_{2}}(G)=2 m^{2}-\frac{1}{2} M_{1}(G) .
$$

Theorem 4 ([31]). Let $G$ be a graph. Then for any $v, v^{\prime} \in V(G)$,

$$
\frac{1}{2} d_{S(G)}\left(v, v^{\prime}\right)=d_{T(G)}\left(v, v^{\prime}\right)=d_{R(G)}\left(v, v^{\prime}\right)=d_{Q(G)}\left(v, v^{\prime}\right)-1=d_{G}\left(v, v^{\prime}\right) .
$$

Theorem 5 ([31]). Let $G$ be a graph. Then for any $e, e^{\prime} \in E(G)$,

$$
\frac{1}{2} d_{S(G)}\left(e, e^{\prime}\right)=d_{T(G)}\left(e, e^{\prime}\right)=d_{R(G)}\left(e, e^{\prime}\right)-1=d_{Q(G)}\left(e, e^{\prime}\right)=d_{L(G)}\left(e, e^{\prime}\right) .
$$

1 WIENER, HYPER WIENER, AND DEGREE DISTANCE INDICES FOR F-PRODUCT OF GRAPHS

In this section, we consider $F \in\{S, Q, R, T\}$, and compute the Wiener, hyper Wiener, and degree distance indices for $F$-product of two connected graphs $G_{1}$ and $G_{2}$. Let $\left|V\left(G_{i}\right)\right|=n_{i}$, and $\left|E\left(G_{i}\right)\right|=\varepsilon_{i}$ for $i=1,2$. Throughout this section we assume that

$\sum_{1}:=\sum_{\{(u, x),(v, y)\} \subseteq V(G), u=v \in V\left(G_{1}\right), x y \in E\left(G_{2}\right)}$,

$\sum_{2}:=\sum_{\{(u, x),(v, y)\} \subseteq V(G), u=v \in V\left(G_{1}\right), x y \notin E\left(G_{2}\right),}$

$\sum_{3}:=\sum_{\{(u, x),(v, y)\} \subseteq V(G), u=v \in V\left(F\left(G_{1}\right)\right)-V\left(G_{1}\right), x, y \in V\left(G_{2}\right)}$, and

$\sum_{4}:=\sum_{\{(u, x),(v, y)\} \subseteq V(G), u \neq v, x, y \in V\left(G_{2}\right)}$. 


\subsection{Wiener index and hyper Wiener index}

Theorem 6. Let $G_{1}$ and $G_{2}$ be two connected graphs, and let $G=G_{1}\left[G_{2}\right]_{F}$. Then

$$
W(G)=n_{1} n_{2}\left(n_{2}-1\right)-n_{1} \varepsilon_{2}+\varepsilon_{1} n_{2}\left(n_{2}-1\right)+n_{2}^{2} W\left(F\left(G_{1}\right)\right) .
$$

Proof. By Theorem 1, we have

$$
\begin{aligned}
W(G) & =\sum_{\{(u, x),(v, y)\} \subseteq V(G)} d_{G}((u, x),(v, y)) \\
& =\sum_{1} 1+\sum_{2} 2+\sum_{3} 2+\sum_{4} d_{F\left(G_{1}\right)}(u, v) \\
& =n_{1} \varepsilon_{2}+2 n_{1}\left(\frac{n_{2}\left(n_{2}-1\right)}{2}-\varepsilon_{2}\right)+2 \varepsilon_{1} \frac{n_{2}\left(n_{2}-1\right)}{2}+n_{2}^{2} W\left(F\left(G_{1}\right)\right) \\
& =n_{1} n_{2}\left(n_{2}-1\right)-n_{1} \varepsilon_{2}+\varepsilon_{1} n_{2}\left(n_{2}-1\right)+n_{2}^{2} W\left(F\left(G_{1}\right)\right) .
\end{aligned}
$$

Theorem 7. Let $G_{1}$ and $G_{2}$ be two connected graphs, and let $G=G_{1}\left[G_{2}\right]_{F}$. Then

$$
W W(G)=-4 n_{1} \varepsilon_{2}+3 n_{2}\left(n_{2}-1\right)\left(n_{1}+\varepsilon_{1}\right)+n_{2}^{2} W W\left(F\left(G_{1}\right)\right) .
$$

Proof. By Theorem 1, we have

$$
\begin{aligned}
W W(G) & =\sum_{\{(u, x),(v, y)\} \subseteq V(G)\}}\left[d_{G}((u, x),(v, y))+d_{G}^{2}((u, x),(v, y))\right] \\
& =\sum_{1} 2+\sum_{2} 6+\sum_{3} 6+\sum_{4}\left[d_{F\left(G_{1}\right)}(u, v)+d_{F\left(G_{1}\right)}^{2}(u, v)\right] \\
& =2 n_{1} \varepsilon_{2}+6 n_{1}\left(\frac{n_{2}\left(n_{2}-1\right)}{2}-\varepsilon_{2}\right)+6 \varepsilon_{1} \frac{n_{2}\left(n_{2}-1\right)}{2}+n_{2}^{2} W W\left(F\left(G_{1}\right)\right) \\
& =-4 n_{1} \varepsilon_{2}+3 n_{2}\left(n_{2}-1\right)\left(n_{1}+\varepsilon_{1}\right)+n_{2}^{2} W W\left(F\left(G_{1}\right)\right) .
\end{aligned}
$$

\subsection{Degree distance index}

\subsubsection{The case $\mathrm{F}=\mathrm{S}$}

Theorem 8 ([26]). If $G_{1}$ and $G_{2}$ are two connected graphs of orders $n_{1}$ and $n_{2}$, respectively, and $G=G_{1}\left[G_{2}\right]_{S}$, then

$$
d((u, x) / G)= \begin{cases}n_{2} d\left(u / G_{1}\right)+d\left(x / G_{2}\right) & \text { if } u \in V\left(G_{1}\right), \\ 2 n_{2} & \text { if } u \in V\left(S\left(G_{1}\right)\right)-V\left(G_{1}\right) .\end{cases}
$$

Theorem 9. Let $G_{i}$ be a connected graph of order $n_{i}$, and size $\varepsilon_{i}$ for $i=1,2$, and let $G=G_{1}\left[G_{2}\right]_{S}$. Then

$$
\begin{aligned}
D D(G) & =2\left(n_{2}-1\right)\left(4 \varepsilon_{1} n_{2}^{2}+\varepsilon_{2} n_{1}\right)-4 n_{2} \varepsilon_{1} \varepsilon_{2}+n_{1} \bar{M}_{1}\left(G_{2}\right)+2 n_{2}^{3} D D\left(G_{1}\right) \\
& +4 n_{2}\left(\varepsilon_{2}-n_{2}^{2}\right) W\left(G_{1}\right)+4 n_{2}^{2}\left(n_{2}^{2}-\varepsilon_{2}\right) W\left(L\left(G_{1}\right)\right)+\left(2 n_{2} \varepsilon_{2}+2 n_{2}^{3}\right) W\left(S\left(G_{1}\right)\right) \\
& +n_{2}^{3} \sum_{u \in V\left(G_{1}\right), v \in V\left(S\left(G_{1}\right)\right)-V\left(G_{1}\right)} d\left(u / G_{1}\right) d_{S\left(G_{1}\right)}(u, v) .
\end{aligned}
$$

Proof. Let $e_{u}$ be the corresponding edge to the new vertex $u$. We deduce from Theorems 1, 2, $3,4,5$ and 8 , that

$$
\begin{aligned}
D D(G) & =\sum_{\{(u, x),(v, y)\} \subseteq V(G)}[d((u, x) / G)+d((v, y) / G)] d_{G}((u, x),(v, y)) \\
& =\sum_{1}[d((u, x) / G)+d((u, y) / G)] \\
& +2 \sum_{2}[d((u, x) / G)+d((u, y) / G)] \\
& +2 \sum_{3}[d((u, x) / G)+d((u, y) / G)] \\
& +\sum_{4}\left[(d((u, x) / G)+d((v, y) / G)) d_{S\left(G_{1}\right)}(u, v)\right]
\end{aligned}
$$


and

$$
\begin{aligned}
& \sum_{1}[d((u, x) / G)+d((u, y) / G)]=\sum_{1}\left[2 n_{2} d\left(u / G_{1}\right)+d\left(x / G_{2}\right)+d\left(y / G_{2}\right)\right] \\
& =4 n_{2} \varepsilon_{2} \varepsilon_{1}+n_{1} M_{1}\left(G_{2}\right) \text {. } \\
& 2 \sum_{2}[d((u, x) / G)+d((u, y) / G)]=2 \sum_{2}\left[2 n_{2} d\left(u / G_{1}\right)+d\left(x / G_{2}\right)+d\left(y / G_{2}\right)\right] \\
& =4 n_{2}^{2} \varepsilon_{1}\left(n_{2}-1\right)-8 n_{2} \varepsilon_{1} \varepsilon_{2}+2 n_{1} \bar{M}_{1}\left(G_{2}\right) \text {. } \\
& 2 \sum_{3}[d((u, x) / G)+d((u, y) / G)]=2 \sum_{3} 4 n_{2}=4 n_{2}^{2} \varepsilon_{1}\left(n_{2}-1\right) . \\
& \sum_{4}\left[(d((u, x) / G)+d((v, y) / G)) d_{S\left(G_{1}\right)}(u, v)\right] \\
& =\sum_{u \neq v, u, v \in V\left(G_{1}\right), x, y \in V\left(G_{2}\right)}\left[n_{2}\left(d\left(u / G_{1}\right)+d\left(v / G_{1}\right)\right)+d\left(x / G_{2}\right)+d\left(y / G_{2}\right)\right] d_{S\left(G_{1}\right)}(u, v) \\
& +\sum_{u \neq v, u, v \in V\left(S\left(G_{1}\right)\right)-V\left(G_{1}\right), x, y \in V\left(G_{2}\right)} 4 n_{2} d_{S\left(G_{1}\right)}(u, v) \\
& +\sum_{u \in V\left(G_{1}\right), v \in V\left(S\left(G_{1}\right)\right)-V\left(G_{1}\right), x, y \in V\left(G_{2}\right)}\left[n_{2} d\left(u / G_{1}\right)+d\left(x / G_{2}\right)+2 n_{2}\right] d_{S\left(G_{1}\right)}(u, v) \\
& =2 n_{2}^{3} D D\left(G_{1}\right)+2 W\left(G_{1}\right)\left(2 M_{1}\left(G_{2}\right)+2 \bar{M}_{1}\left(G_{2}\right)+4 \varepsilon_{2}\right) \\
& +4 n_{2}^{3} \sum_{e_{u}, e_{v} \in V\left(L\left(G_{1}\right)\right)} 2 d_{L\left(G_{1}\right)}\left(e_{u}, e_{v}\right)+n_{2}^{3} \sum_{u \in V\left(G_{1}\right), v \in\left(V\left(S\left(G_{1}\right)\right)-V\left(G_{1}\right)\right)} d\left(u / G_{1}\right) d_{S\left(G_{1}\right)}(u, v) \\
& +\left(2 n_{2} \varepsilon_{2}+2 n_{2}^{3}\right) \sum_{u \in V\left(G_{1}\right), v \in V\left(S\left(G_{1}\right)\right)-V\left(G_{1}\right)} d_{S\left(G_{1}\right)}(u, v) \\
& =2 n_{2}^{3} D D\left(G_{1}\right)+8 \varepsilon_{2} n_{2} W\left(G_{1}\right)+8 n_{2}^{3} W\left(L\left(G_{1}\right)\right) \\
& +n_{2}^{3} \sum_{u \in V\left(G_{1}\right), v \in V\left(S\left(G_{1}\right)\right)-V\left(G_{1}\right)} d\left(u / G_{1}\right) d_{S\left(G_{1}\right)}(u, v) \\
& +\left(2 n_{2} \varepsilon_{2}+2 n_{2}^{3}\right)\left[W\left(S\left(G_{1}\right)\right)-2 W\left(G_{1}\right)-2 W\left(L\left(G_{1}\right)\right)\right] \text {. }
\end{aligned}
$$

Therefore

$$
\begin{aligned}
D D(G) & =2\left(n_{2}-1\right)\left(4 \varepsilon_{1} n_{2}^{2}+\varepsilon_{2} n_{1}\right)-4 n_{2} \varepsilon_{1} \varepsilon_{2}+n_{1} \bar{M}_{1}\left(G_{2}\right)+2 n_{2}^{3} D D\left(G_{1}\right) \\
& +4 n_{2}\left(\varepsilon_{2}-n_{2}^{2}\right) W\left(G_{1}\right)+4 n_{2}^{2}\left(n_{2}^{2}-\varepsilon_{2}\right) W\left(L\left(G_{1}\right)\right)+\left(2 n_{2} \varepsilon_{2}+2 n_{2}^{3}\right) W\left(S\left(G_{1}\right)\right) \\
& +n_{2}^{3} \sum_{u \in V\left(G_{1}\right), v \in V\left(S\left(G_{1}\right)\right)-V\left(G_{1}\right)} d\left(u / G_{1}\right) d_{S\left(G_{1}\right)}(u, v) .
\end{aligned}
$$

\subsubsection{The case $F=R$}

Theorem 10 ([26]). If $G_{1}$ and $G_{2}$ are two connected graphs of orders $n_{1}$ and $n_{2}$, respectively, and let $G=G_{1}\left[G_{2}\right]_{R}$. Then

$$
d((u, x) / G)=\left\{\begin{array}{lll}
2 n_{2} d\left(u / G_{1}\right)+d\left(x / G_{2}\right) & \text { if } u \in V\left(G_{1}\right) \\
2 n_{2} & \text { if } u \in V\left(R\left(G_{1}\right)\right)-V\left(G_{1}\right) .
\end{array}\right.
$$

Theorem 11. Let $G_{i}$ be a connected graph of order $n_{i}$, and size $\varepsilon_{i}$ for $i=1,2$, and let $G=$ $G_{1}\left[G_{2}\right]_{R}$. Then

$$
\begin{aligned}
D D(G) & =2\left(n_{2}-1\right)\left(6 \varepsilon_{1} n_{2}^{2}+\varepsilon_{2} n_{1}\right)-8 n_{2} \varepsilon_{1} \varepsilon_{2}+n_{1}\left(\overline{M_{1}}\left(G_{2}\right)+2 n_{2}^{3} D D\left(G_{1}\right)\right. \\
& \left.+4 \varepsilon_{2} n_{2} W\left(G_{1}\right)+4 \varepsilon_{2}\right)+4 n_{2}^{3}\left[W\left(L\left(G_{1}\right)\right)+\frac{\varepsilon_{1}\left(\varepsilon_{1}-1\right)}{2}\right] \\
& +\left(2 n_{2} \varepsilon_{2}+2 n_{2}^{3}\right)\left[W\left(R\left(G_{1}\right)\right)-W\left(G_{1}\right)-W\left(L\left(G_{1}\right)\right)-\frac{\varepsilon_{1}\left(\varepsilon_{1}-1\right)}{2}\right] \\
& +2 n_{2}^{3} \sum_{u \in V\left(G_{1}\right), v \in V\left(R\left(G_{1}\right)\right)-V\left(G_{1}\right)} d\left(u / G_{1}\right) d_{R\left(G_{1}\right)}(u, v) .
\end{aligned}
$$


Proof. Let $e_{u}$ be the corresponding edge to the new vertex $u$. By Theorems 1, 2, 3, 4, 5 and 10,

and

$$
\begin{aligned}
D D(G) & =\sum_{\{(u, x),(v, y)\} \subseteq V(G)}[d((u, x) / G)+d((v, y) / G)] d_{G}((u, x),(v, y)) \\
& =\sum_{1}[d((u, x) / G)+d((u, y) / G)] \\
& +2 \sum_{2}[d((u, x) / G)+d((u, y) / G)] \\
& +2 \sum_{3}[d((u, x) / G)+d((u, y) / G)] \\
& +\sum_{4}\left[(d((u, x) / G)+d((v, y) / G)) d_{R\left(G_{1}\right)}(u, v)\right]
\end{aligned}
$$

$$
\begin{aligned}
& \sum_{1}[d((u, x) / G)+d((u, y) / G)]=\sum_{1}\left[4 n_{2} d\left(u / G_{1}\right)+d\left(x / G_{2}\right)+d\left(y / G_{2}\right)\right] \\
&=8 n_{2} \varepsilon_{2} \varepsilon_{1}+n_{1} M_{1}\left(G_{2}\right), \\
& 2 \sum_{2}[d((u, x) / G)+d((u, y) / G)]=2 \sum_{2}\left[4 n_{2} d\left(u / G_{1}\right)+d\left(x / G_{2}\right)+d\left(y / G_{2}\right)\right] \\
&=8 n_{2}^{2} \varepsilon_{1}\left(n_{2}-1\right)-16 n_{2} \varepsilon_{1} \varepsilon_{2}+2 n_{1} \bar{M}_{1}\left(G_{2}\right), \\
& 2 \sum_{3}[d((u, x) / G)+d((u, y) / G)]=2 \sum_{3} 4 n_{2}=4 n_{2}^{2} \varepsilon_{1}\left(n_{2}-1\right),
\end{aligned}
$$$$
\sum_{4}\left[(d((u, x) / G)+d((v, y) / G)) d_{R\left(G_{1}\right)}(u, v)\right]
$$$$
=\sum_{u \neq v, u, v \in V\left(G_{1}\right), x, y \in V\left(G_{2}\right)}\left[2 n_{2}\left(d\left(u / G_{1}\right)+d\left(v / G_{1}\right)\right)+d\left(x / G_{2}\right)+d\left(y / G_{2}\right)\right] d_{R\left(G_{1}\right)}(u, v)
$$$$
+\sum_{u \neq v, u, v \in V\left(R\left(G_{1}\right)\right)-V\left(G_{1}\right), x, y \in V\left(G_{2}\right)} 4 n_{2} d_{R\left(G_{1}\right)}(u, v)
$$$$
+\sum_{u \in V\left(G_{1}\right), v \in V\left(R\left(G_{1}\right)\right)-V\left(G_{1}\right), x, y \in V\left(G_{2}\right)}\left[2 n_{2} d\left(u / G_{1}\right)+d\left(x / G_{2}\right)+2 n_{2}\right] d_{R\left(G_{1}\right)}(u, v)
$$$$
=2 n_{2}^{3} D D\left(G_{1}\right)+W\left(G_{1}\right)\left(2 M_{1}\left(G_{2}\right)+2 \bar{M}_{1}\left(G_{2}\right)+4 \varepsilon_{2}\right)
$$$$
+4 n_{2}^{3} \sum_{e_{u}, e_{v} \in V\left(L\left(G_{1}\right)\right)}\left(d_{L\left(G_{1}\right)}\left(e_{u}, e_{v}\right)+1\right)+2 n_{2}^{3} \sum_{u \in V\left(G_{1}\right), v \in V\left(R\left(G_{1}\right)\right)-V\left(G_{1}\right)} d\left(u / G_{1}\right) d_{R\left(G_{1}\right)}(u, v)
$$$$
+\left(2 n_{2} \varepsilon_{2}+2 n_{2}^{3}\right) \sum_{u \in V\left(G_{1}\right), v \in V\left(R\left(G_{1}\right)\right)-V\left(G_{1}\right)} d_{R\left(G_{1}\right)}(u, v)
$$$$
=2 n_{2}^{3} D D\left(G_{1}\right)+4 \varepsilon_{2} n_{2} W\left(G_{1}\right)+4 n_{2}^{3}\left[W\left(L\left(G_{1}\right)\right)+\frac{\varepsilon_{1}\left(\varepsilon_{1}-1\right)}{2}\right]
$$$$
+2 n_{2}^{3} \sum_{u \in V\left(G_{1}\right), v \in V\left(R\left(G_{1}\right)\right)-V\left(G_{1}\right)} d\left(u / G_{1}\right) d_{R\left(G_{1}\right)}(u, v)
$$$$
+\left(2 n_{2} \varepsilon_{2}+2 n_{2}^{3}\right)\left[W\left(R\left(G_{1}\right)\right)-W\left(G_{1}\right)-W\left(L\left(G_{1}\right)\right)-\frac{\varepsilon_{1}\left(\varepsilon_{1}-1\right)}{2}\right] .
$$

Then

$$
\begin{aligned}
D D(G) & =2\left(n_{2}-1\right)\left(6 \varepsilon_{1} n_{2}^{2}+\varepsilon_{2} n_{1}\right)-8 n_{2} \varepsilon_{1} \varepsilon_{2}+n_{1} \overline{M_{1}}\left(G_{2}\right)+2 n_{2}^{3} D D\left(G_{1}\right) \\
& +4 \varepsilon_{2} n_{2} W\left(G_{1}\right)+4 n_{2}^{3}\left[W\left(L\left(G_{1}\right)\right)+\frac{\varepsilon_{1}\left(\varepsilon_{1}-1\right)}{2}\right] \\
& +\left(2 n_{2} \varepsilon_{2}+2 n_{2}^{3}\right)\left[W\left(R\left(G_{1}\right)\right)-W\left(G_{1}\right)-W\left(L\left(G_{1}\right)\right)-\frac{\varepsilon_{1}\left(\varepsilon_{1}-1\right)}{2}\right] \\
& +2 n_{2}^{3} \sum_{u \in V\left(G_{1}\right), v \in V\left(R\left(G_{1}\right)\right)-V\left(G_{1}\right)} d\left(u / G_{1}\right) d_{R\left(G_{1}\right)}(u, v) .
\end{aligned}
$$

\subsubsection{The case $\mathrm{F}=\mathrm{T}$}

Theorem 12 ([26]). If $G_{1}$ and $G_{2}$ are two connected graphs of order $n_{1}$, and $n_{2}$, respectively, and let $T\left(G_{1}\right)$ be the defined graph of $G_{1}$ such that $u$ is the new vertex corresponding to the edge $e_{u}=w w^{\prime}$. Then in graph $G=G_{1}\left[G_{2}\right]_{T}$ we have

$$
d((u, x) / G)=\left\{\begin{array}{lll}
n_{2} d\left(u / G_{1}\right)+d\left(x / G_{2}\right) & \text { if } u \in V\left(G_{1}\right), \\
n_{2} d\left(e_{u}\right) & \text { if } u \in V\left(T\left(G_{1}\right)\right)-V\left(G_{1}\right) .
\end{array}\right.
$$


Theorem 13. Let $G_{i}$ be a connected graph of order $n_{i}$, and size $\varepsilon_{i}$ for $i=1,2$, and let $G=$ $G_{1}\left[G_{2}\right]_{T}$. Then

$$
\begin{aligned}
D D(G) & =2\left(n_{2}-1\right)\left(2 \varepsilon_{1} n_{2}^{2}+\varepsilon_{2} n_{1}\right)-4 n_{2} \varepsilon_{1} \varepsilon_{2}+n_{1} \bar{M}_{1}\left(G_{2}\right)+2 n_{2}^{2}\left(n_{2}-1\right) M_{1}\left(G_{1}\right) \\
& +n_{2}^{3} D D\left(G_{1}\right)+4\left[\varepsilon_{2} n_{2} W\left(G_{1}\right)+W\left(L\left(G_{1}\right)\right)\right]+n_{2}^{3} D D\left(L\left(G_{1}\right)\right) \\
& +2 n_{2} \varepsilon_{2}\left[W\left(T\left(G_{1}\right)\right)-W\left(G_{1}\right)-W\left(L\left(G_{1}\right)\right)\right] \\
& +n_{2}^{3} \sum_{u \in V\left(G_{1}\right), v \in\left(V\left(T\left(G_{1}\right)\right)-V\left(G_{1}\right)\right)}\left[d\left(u / G_{1}\right)+d\left(e_{u}\right)\right] d_{T\left(G_{1}\right)}(u, v) .
\end{aligned}
$$

Proof. Let $e_{u}$ be the corresponding edge to the new vertex $u$. We deduce from Theorems 1, 2, $3,4,5$ and 12 , that

$$
\begin{aligned}
D D(G) & =\sum_{\{(u, x),(v, y)\} \subseteq V(G)}[d((u, x) / G)+d((v, y) / G)] d_{G}((u, x),(v, y)) \\
& =\sum_{1}[d((u, x) / G)+d((u, y) / G)] \\
& +2 \sum_{2}[d((u, x) / G)+d((u, y) / G)] \\
& +2 \sum_{3}[d((u, x) / G)+d((u, y) / G)] \\
& +\sum_{4}\left[(d((u, x) / G)+d((v, y) / G)) d_{T\left(G_{1}\right)}(u, v)\right] .
\end{aligned}
$$

and

$$
\begin{aligned}
& \sum_{1}[d((u, x) / G)+d((u, y) / G)]=\sum_{1}\left[2 n_{2} d\left(u / G_{1}\right)+d\left(x / G_{2}\right)+d\left(y / G_{2}\right)\right] \\
& =4 n_{2} \varepsilon_{2} \varepsilon_{1}+n_{1} M_{1}\left(G_{2}\right) \text {. } \\
& 2 \sum_{2}[d((u, x) / G)+d((u, y) / G)]=2 \sum_{2}\left[2 n_{2} d\left(u / G_{1}\right)+d\left(x / G_{2}\right)+d\left(y / G_{2}\right)\right] \\
& =4 n_{2}^{2} \varepsilon_{1}\left(n_{2}-1\right)-8 n_{2} \varepsilon_{1} \varepsilon_{2}+2 n_{1} \bar{M}_{1}\left(G_{2}\right) \text {. } \\
& 2 \sum_{3}[d((u, x) / G)+d((u, y) / G)]=2 \sum_{3} 2 n_{2} d\left(e_{u}\right) \\
& =2 n_{2}^{2}\left(n_{2}-1\right) M_{1}\left(G_{1}\right) \text {. } \\
& \sum_{4}\left[(d((u, x) / G)+d((v, y) / G)) d_{T\left(G_{1}\right)}(u, v)\right] \\
& =\sum_{u \neq v, u, v \in V\left(G_{1}\right), x, y \in V\left(G_{2}\right)}\left[n_{2}\left(d\left(u / G_{1}\right)+d\left(v / G_{1}\right)\right)+d\left(x / G_{2}\right)+d\left(y / G_{2}\right)\right] d_{T\left(G_{1}\right)}(u, v) \\
& +\sum_{u \neq v, u, v \in V\left(T\left(G_{1}\right)\right)-V\left(G_{1}\right), x, y \in V\left(G_{2}\right)}\left[n_{2}\left(d\left(e_{u}\right)+d\left(e_{v}\right)\right) d_{T\left(G_{1}\right)}(u, v)\right] \\
& +\sum_{u \in V\left(G_{1}\right), v \in V\left(T\left(G_{1}\right)\right)-V\left(G_{1}\right), x, y \in V\left(G_{2}\right)}\left[n_{2} d\left(u / G_{1}\right)+d\left(x / G_{2}\right)+n_{2} d\left(e_{v}\right)\right] d_{T\left(G_{1}\right)}(u, v) \\
& =n_{2}^{3} D D\left(G_{1}\right)+W\left(G_{1}\right)\left(2 M_{1}\left(G_{2}\right)+2 \bar{M}_{1}\left(G_{2}\right)+4 \varepsilon_{2}\right) \\
& +n_{2}^{3} \sum_{u \neq v, u, v \in V\left(T\left(G_{1}\right)\right)-V\left(G_{1}\right)}\left[d\left(e_{u} / L\left(G_{1}\right)\right)+d\left(e_{v} / L\left(G_{1}\right)\right)+4\right] d_{L\left(G_{1}\right)}\left(e_{u}, e_{v}\right) \\
& +n_{2}^{3} \sum_{u \in V\left(G_{1}\right), v \in V\left(T\left(G_{1}\right)\right)-V\left(G_{1}\right)}\left[d\left(u / G_{1}\right)+d\left(e_{u}\right)\right] d_{T\left(G_{1}\right)}(u, v) \\
& +2 n_{2} \varepsilon_{2} \sum_{u \in V\left(G_{1}\right), v \in V\left(T\left(G_{1}\right)\right)-V\left(G_{1}\right)} d_{T\left(G_{1}\right)}(u, v) \\
& =n_{2}^{3} D D\left(G_{1}\right)+n_{2}^{3} D D\left(L\left(G_{1}\right)\right)+4\left[\varepsilon_{2} n_{2} W\left(G_{1}\right)+W\left(L\left(G_{1}\right)\right)\right] \\
& +n_{2}^{3} \sum_{u \in V\left(G_{1}\right), v \in V\left(T\left(G_{1}\right)\right)-V\left(G_{1}\right)}\left[d\left(u / G_{1}\right)+d\left(e_{u}\right)\right] d_{T\left(G_{1}\right)}(u, v) \\
& +2 n_{2} \varepsilon_{2}\left[W\left(T\left(G_{1}\right)\right)-W\left(G_{1}\right)-W\left(L\left(G_{1}\right)\right)\right] \text {. }
\end{aligned}
$$

Hence

$$
\begin{aligned}
D D(G) & =2\left(n_{2}-1\right)\left(2 \varepsilon_{1} n_{2}^{2}+\varepsilon_{2} n_{1}\right)-4 n_{2} \varepsilon_{1} \varepsilon_{2}+n_{1} \bar{M}_{1}\left(G_{2}\right)+2 n_{2}^{2}\left(n_{2}-1\right) M_{1}\left(G_{1}\right) \\
& +n_{2}^{3} D D\left(G_{1}\right)+4\left[\varepsilon_{2} n_{2} W\left(G_{1}\right)+W\left(L\left(G_{1}\right)\right)\right]+n_{2}^{3} D D\left(L\left(G_{1}\right)\right) \\
& +2 n_{2} \varepsilon_{2}\left[W\left(T\left(G_{1}\right)\right)-W\left(G_{1}\right)-W\left(L\left(G_{1}\right)\right)\right] \\
& +n_{2}^{3} \sum_{u \in V\left(G_{1}\right), v \in\left(V\left(T\left(G_{1}\right)\right)-V\left(G_{1}\right)\right)}\left[d\left(u / G_{1}\right)+d\left(e_{u}\right)\right] d_{T\left(G_{1}\right)}(u, v) .
\end{aligned}
$$




\subsubsection{The case $F=Q$}

Theorem 14 ([26]). If $G_{1}$ and $G_{2}$ are two connected graphs of order $n_{1}$ and $n_{2}$, respectively, and let $Q\left(G_{1}\right)$ be the graph obtained from $G_{1}$ by inserting a new vertex into each edge of $G_{1}$, then joining with edges those pairs of new vertices on adjacent edges of $G_{1}$. Suppose that $u$ is the new vertex inserted at the edge $e_{u}=w w^{\prime}$. Then in graph $G=G_{1}\left[G_{2}\right]_{Q}$ we have

$$
d((u, x) / G)=\left\{\begin{array}{lll}
n_{2} d\left(u / G_{1}\right)+d\left(x / G_{2}\right) & \text { if } u \in V\left(G_{1}\right), \\
n_{2} d\left(e_{u}\right) & \text { if } u \in V\left(Q\left(G_{1}\right)\right)-V\left(G_{1}\right) .
\end{array}\right.
$$

Theorem 15. Let $G_{i}$ be a connected graph of order $n_{i}$, and size $\varepsilon_{i}$ for $i=1,2$, and let $G=$ $G_{1}\left[G_{2}\right]_{Q}$. Then

$$
\begin{aligned}
D D(G) & =2\left(n_{2}-1\right)\left(2 \varepsilon_{1} n_{2}^{2}+\varepsilon_{2} n_{1}\right)-4 n_{2} \varepsilon_{1} \varepsilon_{2}+n_{1} \bar{M}_{1}\left(G_{2}\right)+2 n_{2}^{2}\left(n_{2}-1\right) M_{1}\left(G_{1}\right) \\
& +n_{2}^{3}\left(D D\left(G_{1}\right)+M_{1}\left(G_{1}\right)\right)+2 \varepsilon_{2} n_{2}\left(2 W\left(G_{1}\right)+n_{2}\left(n_{2}-1\right)\right)+n_{2}^{3} D D\left(L\left(G_{1}\right)\right) \\
& +4 W\left(L\left(G_{1}\right)\right)+2 n_{2} \varepsilon_{2}\left[W\left(Q\left(G_{1}\right)\right)-W\left(G_{1}\right)-W\left(L\left(G_{1}\right)\right)-\frac{n_{1}\left(n_{1}-1\right)}{2}\right] \\
& +n_{2}^{3} \sum_{u \in V\left(G_{1}\right), v \in V\left(Q\left(G_{1}\right)\right)-V\left(G_{1}\right)}\left[\left(d\left(u / G_{1}\right)+d\left(e_{u}\right)\right) d_{Q\left(G_{1}\right)}(u, v)\right] .
\end{aligned}
$$

Proof. Let $e_{u}$ be the corresponding edge to the new vertex $u$. By Theorems 1, 2, 3, 4, 5 and 14,

$$
\begin{aligned}
D D(G) & =\sum_{\{(u, x),(v, y)\} \subseteq V(G)}[d((u, x) / G)+d((v, y) / G)] d_{G}((u, x),(v, y)) \\
& =\sum_{1}[d((u, x) / G)+d((u, y) / G)]+2 \sum_{2}[d((u, x) / G)+d((u, y) / G)] \\
& +2 \sum_{3}[d((u, x) / G)+d((u, y) / G)] \\
& +\sum_{4}\left[(d((u, x) / G)+d((v, y) / G)) d_{Q\left(G_{1}\right)}(u, v)\right]
\end{aligned}
$$

and

$$
\begin{aligned}
& \sum_{1}[d((u, x) / G)+d((u, y) / G)]=\sum_{1}\left[2 n_{2} d\left(u / G_{1}\right)+d\left(x / G_{2}\right)+d\left(y / G_{2}\right)\right] \\
& =4 n_{2} \varepsilon_{2} \varepsilon_{1}+n_{1} M_{1}\left(G_{2}\right) \text {. } \\
& 2 \sum_{2}[d((u, x) / G)+d((u, y) / G)]=2 \sum_{2}\left[2 n_{2} d\left(u / G_{1}\right)+d\left(x / G_{2}\right)+d\left(y / G_{2}\right)\right] \\
& =4 n_{2}^{2} \varepsilon_{1}\left(n_{2}-1\right)-8 n_{2} \varepsilon_{1} \varepsilon_{2}+2 n_{1} \bar{M}_{1}\left(G_{2}\right) \text {. } \\
& 2 \sum_{3}[d((u, x) / G)+d((u, y) / G)]=2 \sum_{3} 2 n_{2} d\left(e_{u}\right)=2 n_{2}^{2}\left(n_{2}-1\right) M_{1}\left(G_{1}\right) . \\
& \sum_{4}\left[(d((u, x) / G)+d((v, y) / G)) d_{Q\left(G_{1}\right)}(u, v)\right] \\
& =\sum_{u \neq v, u, v \in V\left(G_{1}\right), x, y \in V\left(G_{2}\right)}\left[n_{2}\left(d\left(u / G_{1}\right)+d\left(v / G_{1}\right)\right)+d\left(x / G_{2}\right)+d\left(y / G_{2}\right)\right] d_{Q\left(G_{1}\right)}(u, v) \\
& +\sum_{u \neq v, u, v \in V\left(Q\left(G_{1}\right)\right)-V\left(G_{1}\right), x, y \in V\left(G_{2}\right)}\left[n_{2}\left(d\left(e_{u}\right)+d\left(e_{v}\right)\right) d_{Q\left(G_{1}\right)}(u, v)\right] \\
& +\sum_{u \in V\left(G_{1}\right), v \in V\left(Q\left(G_{1}\right)\right)-V\left(G_{1}\right), x, y \in V\left(G_{2}\right)}\left[n_{2} d\left(u / G_{1}\right)+d\left(x / G_{2}\right)+n_{2} d\left(e_{v}\right)\right] d_{Q\left(G_{1}\right)}(u, v) \\
& =n_{2}^{3}\left(D D\left(G_{1}\right)+M_{1}\left(G_{1}\right)\right)+\left(W\left(G_{1}\right)+\frac{n_{2}\left(n_{2}-1\right)}{2}\right)\left(2 M_{1}\left(G_{2}\right)+2 \bar{M}_{1}\left(G_{2}\right)+4 \varepsilon_{2}\right) \\
& +n_{2}^{3} \sum_{u \neq v, u, v \in V\left(T\left(G_{1}\right)\right)-V\left(G_{1}\right)}\left[d\left(e_{u} / L\left(G_{1}\right)\right)+d\left(e_{v} / L\left(G_{1}\right)\right)+4\right] d_{L\left(G_{1}\right)}\left(e_{u}, e_{v}\right) \\
& +n_{2}^{3} \sum_{u \in V\left(G_{1}\right), v \in V\left(Q\left(G_{1}\right)\right)-V\left(G_{1}\right)}\left[\left(d\left(u / G_{1}\right)+d\left(e_{v}\right)\right) d_{Q\left(G_{1}\right)}(u, v)\right] \\
& +2 n_{2} \varepsilon_{2} \sum_{u \in V\left(G_{1}\right), v \in V\left(Q\left(G_{1}\right)\right)-V\left(G_{1}\right)} d_{Q\left(G_{1}\right)}(u, v) \\
& =n_{2}^{3}\left(D D\left(G_{1}\right)+M_{1}\left(G_{1}\right)\right)+2 \varepsilon_{2} n_{2}\left(2 W\left(G_{1}\right)+n_{2}\left(n_{2}-1\right)\right)+n_{2}^{3} D D\left(L\left(G_{1}\right)\right)+4 W\left(L\left(G_{1}\right)\right) \\
& +n_{2}^{3} \sum_{u \in V\left(G_{1}\right), v \in V\left(Q\left(G_{1}\right)\right)-V\left(G_{1}\right)}\left[\left(d\left(u / G_{1}\right)+d\left(e_{u}\right)\right) d_{Q\left(G_{1}\right)}(u, v)\right] \\
& +2 n_{2} \varepsilon_{2}\left[W\left(Q\left(G_{1}\right)\right)-W\left(G_{1}\right)-W\left(L\left(G_{1}\right)\right)-\frac{n_{1}\left(n_{1}-1\right)}{2}\right] \text {. }
\end{aligned}
$$


Hence,

$$
\begin{aligned}
D D(G) & =2\left(n_{2}-1\right)\left(2 \varepsilon_{1} n_{2}^{2}+\varepsilon_{2} n_{1}\right)-4 n_{2} \varepsilon_{1} \varepsilon_{2}+n_{1} \bar{M}_{1}\left(G_{2}\right)+2 n_{2}^{2}\left(n_{2}-1\right) M_{1}\left(G_{1}\right) \\
& +n_{2}^{3}\left(D D\left(G_{1}\right)+M_{1}\left(G_{1}\right)\right)+2 \varepsilon_{2} n_{2}\left(2 W\left(G_{1}\right)+n_{2}\left(n_{2}-1\right)\right)+n_{2}^{3} D D\left(L\left(G_{1}\right)\right) \\
& +4 W\left(L\left(G_{1}\right)\right)+2 n_{2} \varepsilon_{2}\left[W\left(Q\left(G_{1}\right)\right)-W\left(G_{1}\right)-W\left(L\left(G_{1}\right)\right)-\frac{n_{1}\left(n_{1}-1\right)}{2}\right] \\
& +n_{2}^{3} \sum_{u \in V\left(G_{1}\right), v \in V\left(Q\left(G_{1}\right)\right)-V\left(G_{1}\right)}\left[\left(d\left(u / G_{1}\right)+d\left(e_{u}\right)\right) d_{Q\left(G_{1}\right)}(u, v)\right] .
\end{aligned}
$$

\section{REFERENCES}

[1] Aringhieri R., Hansen P., Malucelli F. A linear algorithm for the hyper-Wiener index of chemical trees. J. Chem. Inf. Comput. Sci. 2001, 41, 958-963. doi:10.1021/ci0001536

[2] Ashrafi A., Došlić T., Hamzeh A. The Zagreb coindices of graph operations. Discrete Appl. Math. 2010, 158, 1571-1578.

[3] Basavanagoud B., Patil S. A note on hyper-zagreb index of graph operations. Iran. J. Math. Chem. 2016, 7, 89-92. doi:10.22052/IJMC.2016.12405

[4] Borovicanin B., Das K.C., Furtula B., Gutman I. Bounds for Zagreb indices. MATCH Commun. Math. Comput. Chem. 2017, 78, 17-100.

[5] Das K.C., Gutman I. Some properties of the second Zagreb index. MATCH Commun. Math. Comput. Chem. 2004, 52, 103-112.

[6] Das K.C., Gutman I. The first Zagreb index 30 years after. MATCH Commun. Math. Comput. Chem. 2004, 50, 83-92.

[7] Dehgardi N., Sheikholeslami S.M., Soroudi M. On the leap Zagreb indices of graphs. (submitted).

[8] Diudea M. V., Parv B. Molecular topology. 25. HypernïSWiener index of dendrimers. J. Chem. Inf. Comput. Sci. 1995, 35, 1015-1018.

[9] Dobrynin A. A., Kochetova A. A. Degree Distance of a Graph: A Degree Analogue of the Wiener Index. J. Chem. Inf. Comput. Sci. 1994, 34, 1082-1086.

[10] Došlić T. Vertex-weighted Wiener polynomials for composite graphs. Ars Math. Contemp. 2008, 1, 66-80.

[11] Eliasi M., Iranmanesh A., Gutman I. Multiplicative versions of first zagreb index. MATCH Commun. Math. Comput. Chem. 2012, 68, 217-230.

[12] Eliasi M., Raeisi G., Taeri B. Wiener index of some graph operations. Discrete Appl. Math. 2012, 160, $1333-1344$.

[13] Gutman I. Multiplicative zagreb indices of trees. Bull. Soc. Math. Banja Luka 2011, 18, 17-23.

[14] Gutman I. Selected Properties of the Schultz Molecular Topological Index. J. Chem. Inf. Comput. Sci. 1994, 34, 1087-1089.

[15] Gutman I., Furtula B., Vukićević K., Popivoda G. On Zagreb indices and coindices. MATCH Commun. Math. Comput. Chem. 2015, 74, 5-16.

[16] Gutman I., Linert W., Lukovits I., Dobrynin A. A. Trees with extremal hyper-Wiener index: Mathematical basis and chemical applications. J. Chem. Inf. Comput. Sci. 1997, 37 (2), 349-354. doi:10.1021/ci960139m

[17] Gutman I., Rucčič B., Trinajstić N., Wilcox C.F. Graph theory and molecular orbitals. XII. acyclic polyenes. J. Chem. Phys. 1975, 62, 3399-3405. doi:10.1063/1.430994

[18] Gutman I., Trinajsti N. Graph theory and molecular orbitals. total $\pi$-electron energy of alternant hydrocarbons. Chem. Phys. Lett. 17 (1972), 535-538. doi:10.1016/0009-2614(72)85099-1

[19] Hosoya H. Topological index. A newly proposed quantity characterizing the topological nature of structural isomers of saturated hydrocarbons. Bull. Chem. Soc. Jpn. 1971, 4, 2332-2339. 
[20] Ilić A., Zhou B. On reformulated Zagreb indices. Discrete Appl. Math. 2012, 160 (3), 204-209. doi:10.1016/j.dam.2011.09.021

[21] Khalifeh M.H., Yousefi-Azari H., Ashrafi A.R. The hyper-Wiener index of graph operations. Comput. Math. Appl. 2008, 56, 1402-1407.

[22] Klavžar S., Žigert P., Gutman I. An algorithm for the calculation of the hyper-Wiener index of benzenoid hydrocarbons. MATCH Commun. Math. Comput. Chem. 2000, 24, 229-233.

[23] Lukovits I. Formulas for the hyper-Wiener index of trees. J. Chem. Inf. Comput. Sci. 1994, 34, 1079-1081.

[24] Miličević A., Nikolić S., Trinajstić N. On reformulated zagreb indices. Mol Divers. 2004, 8 (4), $393-399$. doi:10.1023/b:modi.0000047504.14261.2a

[25] Pattabiraman K., Vijayaragavan M. Hyper zagreb indices and its coindices of graphs. Bull. Int. Math. Virtual Inst. 2017, 7, 31-41.

[26] Sarala D., Deng H., Ayyaswamy S.K., Balachandran S. The Zagreb indices of graphs based on four new operations related to the lexicographic product. Appl. Math. Comput. 2017, 309, 156-169.

[27] Wiener H. Structural determination of paraffin boiling points. J. Amer. Chem. Soc. 1947, 69, 17-20.

[28] Xu K., Das K.C. Trees, unicyclic, and bicyclic graphs extremal with respect to multiplicative sum zagreb index. MATCH Commun. Math. Comput. Chem. 2012, 68, 257-272.

[29] Xu K., Das K.C., Tang K. On the multiplicative zagreb coindex of graphs. Opuscula Math. 2013, 33, $191-204$.

[30] Xu K., Hua H. A unified approach to extremal multiplicative zagreb indices for trees, unicyclic and bicyclic graphs. MATCH Commun. Math. Comput. Chem. 2012, 68, 241-256.

[31] Yan W., Yang B-Y., Yeh Y-N. The behavior of Wiener indices and polynomials of graphs under five graph decorations. MATCH Commun. Appl. Math. Lett. 2007, 20, 290-295.

Received 18.05.2018

Аегарді Н, Шейхолесламі С.М., Сороуді М. Аеякі дистаниійні індекси графів, що грунтуються на иотирьох нових операціях, які відносяться до лексикографіиного добутку // Карпатські матем. публ. - 2019. - Т.11, №2. - С. 258-267.

Аля (молекулярного) графу індекс Вінера, гіпервінерівський індекс і індекс степеневої відстані визначаються як $W(G)=\sum_{\{u, v\} \subseteq V(G)} d_{G}(u, v), W W(G)=W(G)+\sum_{\{u, v\} \subseteq V(G)} d_{G}(u, v)^{2}$ i $D D(G)=\sum_{\{u, v\} \subseteq V(G)} d_{G}(u, v)(d(u / G)+d(v / G))$ відповідно. $d(u / G)$ позначає степінь вершини $u$ в $G$ і $d_{G}(u, v)$ — відстань між двома вершинами $u$ і $v$ в графі $G$. У цій статті ми вивчаємо індекс Вінера, гіпервінерівський індекс і індекс степеневої відстані у графрах, що грунтуються на чотирьох нових операціях, які відносяться до лексикографічного добутку, підроздільності та тотального графу.

Ключові слова і фрази: індекс Вінера, індекс степеневої відстані, гіпервінерівський індекс, лексикографічний добуток, підроздільність, тотальний граф. 